\title{
Pure primary signet ring cell carcinoma of the bladder: atypical case and review of the literature
}

\begin{abstract}
Primary signet-ring cell carcinoma is a rare and aggressive histological variant of primary bladder adenocarcinoma with a usually delayed diagnosis and poor prognosis. No standard treatment modality exists for this tumor till now because of its rarity. We present an atypical case of pure primary signet-ring cell carcinoma of the bladder, without obvious bladder mass on CT scan. The patient underwent a radical cystectomy with ileal conduit, and adjuvant chemotherapy (Gemcitabine/ Carboplatin).
\end{abstract}

Keywords: adenocarcinoma, signet ring cell, urinary bladder

\author{
Volume I Issue 3 - 2014 \\ Khalil Armache,' Jawad Feghali,' Ali Bourgi, ${ }^{2}$ \\ Anthony Kallas Chemaly, ${ }^{2}$ Georges Aftimos, ${ }^{3}$ \\ Maroun Moukarzel ${ }^{2}$ \\ 'Department of Urology, Centre Hospitalier du Nord, Saint- \\ Joseph University, Zgharta, Lebanon \\ ${ }^{2}$ Department of Urology, Hotel-Dieu de France Hospital, Saint- \\ Joseph University, Beirut, Lebanon \\ ${ }^{3}$ Department of Pathology, Centre Hospitalier du Nord, Saint- \\ Joseph University, Zgharta, Lebanon
}

Correspondence: Anthony Kallas Chemaly, Department of Urology, Hotel-Dieu de France Hospital, Saint-Joseph University, Beirut Achrafieh, Bvd Alfred Naccache, PO BOX 166830 , Lebanon, Tel 0096/3551326,

Email anthony_chemaly@yahoo.com
Abbreviations: PSRCC, primary signet-ring cell carcinoma; GI, gastro intestinal; CEA, carcinoembryonic antigen; CK, cyto keratin

\section{Introduction}

Bladder adenocarcinoma is a rare histological subtype of bladder cancers, with variant subtypes including pure primary signet-ring cell carcinoma (PSRCC) which accounts for only $0.5-2 \%$ of all primary neoplasms of the bladder. ${ }^{1}$ The first two cases were reported by Saphir. ${ }^{2}$ A review of the literature reveals no more than 100 published cases. The prognosis for patients with SRC is poor, attributed to presentation at advanced stages. Diagnosis of bladder SRC can be challenging, both as it requires an extensive work-up to rule out more common metastatic adenocarcinomas, and comprehensive studies detailing the immunohistochemical profile of these tumors are understandably lacking. ${ }^{3}$ Current treatment for this malignancy is often based on experiences gained from single institutional case series. Based on the aggressive natural history of PSRCC, treatment often features aggressive surgery in conjunction with chemotherapy and/ or radiotherapy. ${ }^{4}$ We present one case of an atypical pure primary signet-ring cell carcinoma of the bladder, and a brief review of the literature.

\section{Case description}

A 66-year-old man was admitted after experiencing painless gross hematuria for a month. The patient's medical history was significant for diabetes and coronary artery bypass surgery 20 years ago. The familial history was unremarkable. The physical exam was normal. Laboratory data were within the normal ranges except for many red blood cells on urinalysis. Pelvis Ultrasound just revealed a thickening of the bladder wall. A CT-scan of abdomen and pelvis showed no obvious bladder mass or distant metastases, but only a diffuse thickening of the bladder wall with bilateral enlargement of the inguinal and iliac lymph nodes (Figure 1). On cystoscopy, multiple small sessile tumors were found in the left bladder wall, base and dome. A transurethral resection was performed. Pathology reports showed a signet ring cell subtype of bladder adenocarcinoma infiltrating the lamina propria and muscular is (stag pT2). We performed a gastroduodenoscopy, colonoscopy and CEA marker level. The normal findings excluded an extravesical primary tumor site. The tumor was then considered to be a primary signet ring cell carcinoma of the bladder. The patient was operated with a radical cystoprostatectomy with an ileal conduit and bilateral pelvic lymphadenectomy. We should mention that a frozen pelvis was found during the surgical operation. Routine pathology examination of the surgical specimen revealed a poorly differentiated adenocarcinoma of the bladder composed of signet ring cells with an abundant mucin pool with invasion of the peri vesical fat (Figure 2). Right ureteral margin was tumor free although left ureteral margin was initially focally reached by tumor. The histopathological staging was pT3bN1M0. Immunohistilogical studies had shown expression of cytokeratin 7, CEA and CA 19-9 but not CD-X2 (Figure 3). The patient was discharged on the $8^{\text {th }}$ day. Postoperative adjuvant chemotherapy with Carboplatin and Gemcitabine combination was started. The patient received six cycles of chemotherapy, but died six months later from a heart attack.

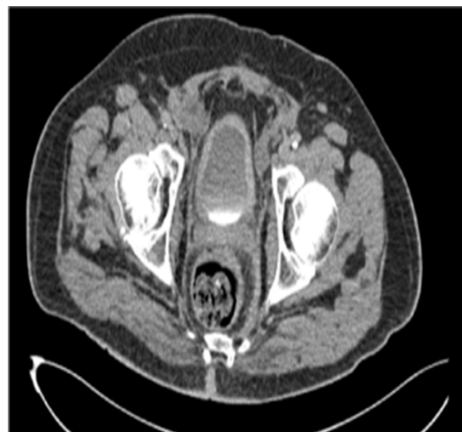

Figure I Abdominopelvic computed tomography showing a diffuse thickening of the urinary bladder. 


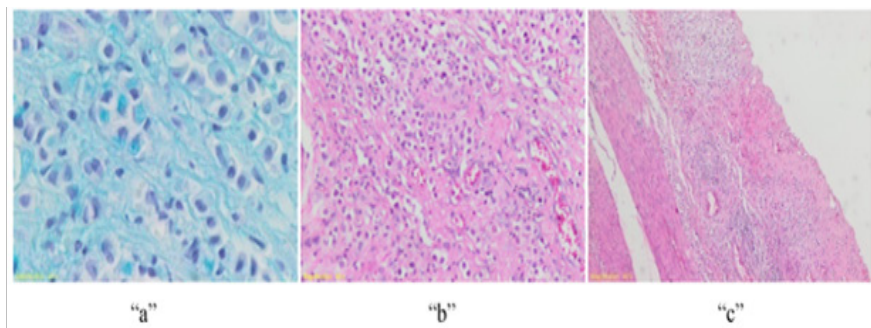

Figure 2 Multiple signet-ring cells located in the lamina propria with overlying transitional cell epithelium (Alcian blue stain in "a" and Hematoxilin-eosin $(\mathrm{HE})$ in "b" and "c").

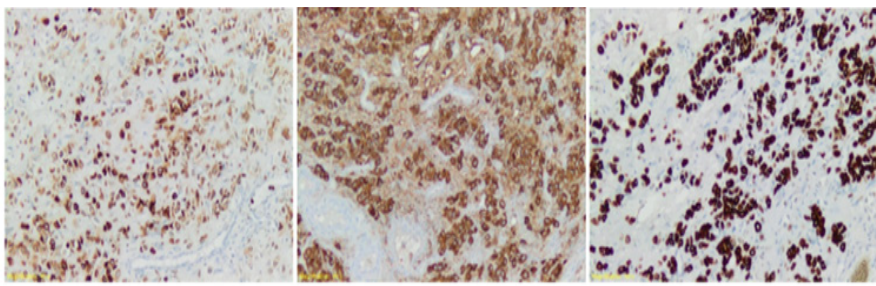

CEA

CA 19.9

CK 7

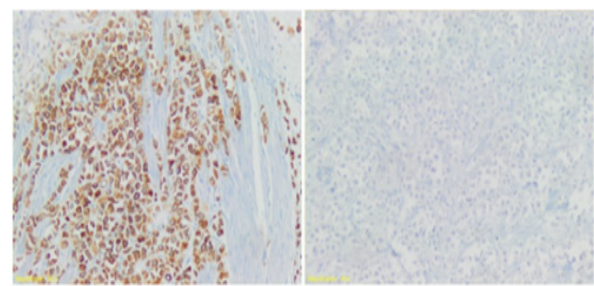

$\mathrm{CD} \times 2$

CK 20

Figure 3 Immunohistochemical findings: IHC positive for CEA, CA 19-19, CK 7, CK 20; negative for CD X2.

\section{Discussion}

PSRCCs are a variant of primary bladder adenocarcinomas, which collectively account for $0.5-2 \%$ of all malignant bladder tumors. ${ }^{3}$ It affects elderly patients in their seventies with a male predominance. ${ }^{2}$ This neoplasia in only rarely found in a histologically pure form. In the majority of cases the atypical elements which characterize it are mixed with the gland-like and papillary structures of the adenocarcinoma and with foci of transitional or pavimentous carcinoma. The origin is probably metaplastic. These carcinoma are generally invasive, scarcely differentiated and often cause linitis plastica of the bladder. The PSRCC is histologically characterized by atypical epithelial elements with abundant, vacuolised cytoplasma. The nuclei are hyper chromic and eccentric, thus representing the typical appearance of signet-ring cells. ${ }^{5}$ The identification of individual cells displaying a signet-ring morphology is important for establishing a pathologic diagnosis ; in fact, Thomas et al., ${ }^{6}$ found significant correlations between the percentage of tumor containing signet-ring cells and both the presence of adverse disease features (unresectability, lymph node involvement) and overall survival. Bladder dome is the site of privilege for adenocarcinoma, however this can also be found in any other regions of the bladder. ${ }^{6}$ Signet-ring cells can also be found in adenocarcinomas of urachus, such tumor origin should be excluded. In our case we were able to exclude an urachal tumor especially that the tumors were found in the lateral wall, base and dome on cystoscopy, and that histology did not show sharp demarcations separating the tumor and the normal bladder surface epithelium. Regardless of site, multiple histologic subtypes of bladder adenocarcinoma exist and include colonic-type adenocarcinoma, mucinous adenocarcinoma, signet-ring cell carcinoma, clear cell carcinoma, hepatoid carcinoma, and non specific adenocarcinoma, as well as mixed forms. ${ }^{8}$

A careful distinction between primary and secondary adenocarcinomas of the bladder (affecting the bladder by direct extension or metastatic spread) is important, because of different therapeutic strategies. Additional evaluations should be performed in order to exclude metastasis, due to histological similarities between primary signet-ring cell carcinoma of the bladder and that of other sites (GI tract, prostate, lung, breast). ${ }^{9}{ }^{10}$ In our case, the gastrointestinal tract evaluation consisted of an esogastroduodenoscopy and colonoscopy, which were normal. Concerning serum tumor markers, several studies reported elevation of the carcinoembryonic antigen (CEA) in primary signet-ring cell bladder adenocarcinoma. In their study on signet-ring cell carcinomas, Yamamoto et al., ${ }^{9}$ reported normal postoperative values of CEA, and showed a correlation between an increase in the level of this serum marker is and disease progression. In our case, no elevation in the level of serum CEA was noted.

Indeed, the distinction between primary adenocarcinomas of the bladder and those secondarily involving the bladder either by direct extension or metastatic spread has been the focus of many immunohistochemical studies. ${ }^{11}$ Specifically, most markers examined have concentrated on the differentiation between colon carcinoma and colonic-type bladder adenocarcinoma because of the morphologic similarity between these 2 entities. Of numerous markers examined, including cytokeratin (CK) 7, CK20, villin-1, CDX-2, $\beta$-catenin, and others, absence of CDX-2 and villin-1 expression appears to be the most robust markers in the determination of primary bladder origin. ${ }^{12}$ However, the utility of these markers in other variants of bladder adenocarcinoma is unclear, with studies from other anatomical locations suggesting that the immunohistochemical profiles of signet-ring cell carcinoma, for example, may vary from more typical adenocarcinomas arising at the same site. ${ }^{13}$

PRSCC has usually a poor prognosis is as it is not diagnosed at an early stage ${ }^{2}$ where $\mathrm{CT}$ does not show obvious bladder mass. To our knowledge, our case is unique by the fact that there are no clear bladder mass or distant metastases shown on the CT scan. Only diffuse thickening of the bladder with inguinal and iliac lymph node enlargement was noted. A reason why more alert and attention should be taken toward such atypical cases in order not to misdiagnose any PSRCC of the urinary bladder. Treatment modalities for primary signet ring cell carcinomas are multiple, and include surgery (transurethral resection of the bladder tumor, radical cystectomy), chemotherapy, and/or radiotherapy. Erdogru et al., ${ }^{14}$ showed that radical cystectomy is the best available surgical option for patients. In fact, only one case was reported on cystectomy with orthotopic neobladder for the treatment of primary signet-ring cell carcinoma of bladder. ${ }^{15}$

A unique and successful treatment was reported by Hirano et al who used intra-arterial chemotherapy alone. ${ }^{16}$ Radiotherapy and chemotherapy alone have had limited success and are usually used as adjuvant therapy after surgery. Unfortunately, there is no standard chemotherapy regimen available for this bladder adenocarcinoma subtype. Our case is a pure PSRCC with no mass on imaging, treated with radical cystectomy with ileal conduit followed post-operatively by Cisplatin/Gemcitabine chemotherapy regimen. This regimen is usually used for the treatment of transitional carcinoma of the bladder. To our knowledge, in addition to its atypical presentation, our case is the second case in the literature treated with this chemotherapy regimen. 


\section{Conclusion}

Primary signet-ring cell carcinoma is an aggressive and unusual form of the primary bladder adenocarcinoma with dismal outcomes for most patients, related to the advanced stage of disease at presentation, the possible poor correlation between diagnostic imaging and tumor stage, the lack of effective systemic therapy, and the poorly defined treatment modalities. The prognosis is only improved with early diagnosis and establishment of standard and effective therapy regimens.

\section{Acknowledgements}

None.

\section{Conflict of interest}

The author declares no conflict of interest.

\section{References}

1. Thomas DG, Ward AM, Williams JL. A study of 52 cases of adenocarcinoma of the bladder. Br J Urol. 1971;43(1):4-15.

2. Saphir O. Signet-ring cell carcinoma of the urinary bladder. Am J Pathol. 1955;31(2):223-231.

3. Roy S, Parwani AV. Adenocarcinoma of the urinary bladder. Arch Pathol Lab Med. 2011;135(12):1601-1605.

4. Reddy K, Moore J, Girdler B, et al. Multidisciplinary management of primary signet-ring cell adenocarcinoma of the urinary bladder and the specific role of adjuvant radiotherapy. Journal of Radiation Oncology. 2013;2(2):1-6.

5. Piana P, A Giammo. Primary signet-ring-cell carcinoma of the bladder. Description of a case and review of the literature. Minerva Urol Nefrol. 1997;49(3):161-164.

6. Thomas AA, Stephenson AJ, Campbell SC, et al. Clinicopathologic features and utility of immunohistochemical markers in signet-ring cell adenocarcinoma of the bladder. Hum Pathol. 2009;40(1):108-116.
7. Sunghwan J, Soojin J, Kweonsik M, et al. Primary signet ring cell carcinoma of the urinary bladder. Korean J Urol. 2009;50(2):188-191.

8. Singh I, Ravi Prasad. Primary Urachal Mucinous Adenocarcinoma of the Urinary Bladder. J Clin Diagn Res. 2013;7(5):911-913.

9. Yamamoto S, Ito T, Akiyama A, et al. Primary signet-ring cell carcinoma of the urinary bladder including renal failure. Int J Urol. 2001;8(4):190 193.

10. Krichen Makni S, Ellouz S, Khabir A, et al. Primary signet ring cell carcinoma of urinary bladder. A case report. Cancer Radiother. 2005;9(5):322-324.

11. Osunkoya AO, Epstein JI. Primary mucin-producing urothelial-type adenocarcinoma of prostate: report of 15 cases. Am J Surg Pathol. 2007;31(9):1323-1329.

12. Suh N, Yang XJ, Tretiakova MS, et al. Value of CDX2, villin, and alpha-methylacyl coenzyme A racemase immunostains in the distinction between primary adenocarcinoma of the bladder and secondary colorectal adenocarcinoma. Mod Pathol. 2005;18(9):1217-1222.

13. Tsuta K, Ishii G, Nitadori J, et al. Comparison of the immunophenotypes of signet-ring cell carcinoma, solid adenocarcinoma with mucin production, and mucinous bronchioloalveolar carcinoma of the lung characterized by the presence of cytoplasmic mucin. J Pathol. 2006;209(1):78-87.

14. Erdogru T, Kilicaslan I, Esen T, et al. Primary signet ring cell carcinoma of the urinary bladder: review of the literature and report of two cases. Urol Int. 1995;55(1):34-37.

15. Fujita M, Otoshi T, Kobayashi K, et al. Primary signet-ring cell carcinoma of the urinary bladder: a case report. Hinyokika kiyo. 2009;55(9):579-582.

16. Hirano Y, Suzuki K, Fujita K, et al. Primary signet ring cell carcinoma of the urinary bladder successfully treated with intra-arterial chemotherapy alone. Urology. 2002;59(4):601. 